University of Wollongong

Research Online

Australian Institute for Innovative Materials -

Papers

Australian Institute for Innovative Materials

$1-1-2018$

Carbon nanotube-induced phase and stability engineering: A strained cobalt-doped WSe2/MWNT heterostructure for enhanced hydrogen evolution reaction

\author{
Guoyuan Zhang \\ Zhengzhou University \\ Xiaoli Zheng \\ Zhengzhou University \\ Qun Xu \\ Zhengzhou University, qunxu@zzu.edu.cn \\ Jianan Zhang \\ Zhengzhou University \\ Wei Liu \\ Zhengzhou University
}

See next page for additional authors

Follow this and additional works at: https://ro.uow.edu.au/aiimpapers

Part of the Engineering Commons, and the Physical Sciences and Mathematics Commons

Research Online is the open access institutional repository for the University of Wollongong. For further information contact the UOW Library: research-pubs@uow.edu.au 


\title{
Carbon nanotube-induced phase and stability engineering: A strained cobalt- doped WSe2/MWNT heterostructure for enhanced hydrogen evolution reaction
}

\author{
Abstract \\ Efficient evolution of hydrogen with inexpensive, highly active and long-term stable catalysts in a wide $\mathrm{pH}$ \\ range holds tremendous promise for clean energy. Here, we report the synthesis of a cobalt-doped \\ tungsten selenide/multiwalled carbon nanotube (Co-WSe 2 /MWNT) heterostructure for the first time, \\ wherein the MWNTs act as an efficient topological template for the nucleation and growth of curved Co- \\ WSe 2 nanosheets with abundant accessible active sites. Furthermore, we demonstrate that the MWNT \\ induced curvature strain not only promotes the predominant 1T phase of active Co-WSe 2 , but also \\ enhances its anti-oxidative stability. Further study indicates that the as-prepared catalyst exhibits efficient \\ electrocatalytic HER in both acidic and alkaline environments with high activity and long-term stability. \\ Disciplines \\ Engineering | Physical Sciences and Mathematics

\section{Publication Details} \\ Zhang, G., Zheng, X., Xu, Q., Zhang, J., Liu, W. \& Chen, J. (2018). Carbon nanotube-induced phase and \\ stability engineering: A strained cobalt-doped WSe2/MWNT heterostructure for enhanced hydrogen \\ evolution reaction. Journal of Materials Chemistry A, 6 (11), 4793-4800.

\section{Authors} \\ Guoyuan Zhang, Xiaoli Zheng, Qun Xu, Jianan Zhang, Wei Liu, and Jun Chen
}




\title{
Carbon nanotube-induced phase and stability engineering: a strained cobalt-doped WSe 2 MWNT heterostructure for enhanced hydrogen evolution reaction†
}

\author{
Guoyuan Zhang, $\ddagger^{\mathrm{a}}$ Xiaoli Zheng, (D) $\ddagger^{\mathrm{a}}$ Qun Xu, (D) *a Jianan Zhang, (D) ${ }^{\mathrm{a}}$ Wei Liu \\ and Jun Chen ${ }^{\mathrm{b}}$
}

Efficient evolution of hydrogen with inexpensive, highly active and long-term stable catalysts in a wide $\mathrm{pH}$ range holds tremendous promise for clean energy. Here, we report the synthesis of a cobalt-doped tungsten selenide/multiwalled carbon nanotube ( $\mathrm{Co}-\mathrm{WS} \mathrm{e}_{2} / \mathrm{MWNT}$ ) heterostructure for the first time, wherein the MWNTs act as an efficient topological template for the nucleation and growth of curved Co$\mathrm{WSe}_{2}$ nanosheets with abundant accessible active sites. Furthermore, we demonstrate that the MWNT induced curvature strain not only promotes the predominant $1 \mathrm{~T}$ phase of active Co-WSe${ }_{2}$, but also enhances its anti-oxidative stability. Further study indicates that the as-prepared catalyst exhibits efficient electrocatalytic HER in both acidic and alkaline environments with high activity and long-term stability.

\section{Introduction}

The development of earth-abundant, highly active and stable catalysts for the hydrogen evolution reaction (HER) is essential for the implementation of clean and renewable hydrogen energy technologies. ${ }^{1-3}$ Two-dimensional (2D) transition-metal dichalcogenides (TMDs), such as molybdenum (Mo) and tungsten (W) dichalcogenides, have emerged as promising alternatives to the expensive platinum (Pt)-based catalysts for the HER. ${ }^{4-7}$ Unfortunately, the limited active sites, poor conductivity and relatively low stability of TMDs severely restrict their catalytic performances. ${ }^{8}$

To date, substantial efforts have been devoted to optimizing the electrocatalytic properties of TMDs, including maximizing the exposure of the edges, ${ }^{9,10}$ inducing defects/vacancies, ${ }^{11-13}$ heteroatom doping, ${ }^{14-17}$ phase engineering ${ }^{18-21}$ and integration of conductive substrates. ${ }^{22-26}$ Among these strategies, phase engineering of TMDs from the semiconducting $2 \mathrm{H}$ phase to the metallic $1 \mathrm{~T}$ phase has been demonstrated as an effective pathway to enhance the catalytic activity, wherein the metastable $1 \mathrm{~T}$ phase possesses intrinsically higher conductivity and

${ }^{a}$ College of Materials Science and Engineering, Zhengzhou University, Zhengzhou 450052, P. R. China.E-mail: qunxu@zzu.edu.cn

${ }^{b}$ ARC Centre of Excellence for Electromaterials Science, Intelligent Polymer Research Institute, Australian Institute of Innovative Materials, University of Wollongong, Wollongong, NSW 2500, Australia

$\dagger$ Electronic supplementary information (ESI) available. See DOI: 10.1039/c8ta00163d

\$ These authors contributed equally to this work. charge transfer kinetics than the $2 \mathrm{H}$ polymorph. ${ }^{27-29}$ However, the most studied lithium intercalation method for phase transformation is tedious and complicated. ${ }^{21}$ Additionally, 1Tphase TMDs are thermodynamically unstable and may rapidly transform into the $2 \mathrm{H}$ phase at elevated temperatures. ${ }^{18,30}$ Apart from phase engineering, some researchers have reported that metal atom doping of TMDs can enhance the inherent activity of the active sites by altering the $\mathrm{DG}_{\mathrm{H}}$ to be close to $0 \mathrm{eV} \cdot{ }^{14}$ However, a high proportion of the exposed metal atoms are usually thermodynamically metastable with high surface energy and vulnerable to oxidation and instability, thus severely reducing the catalytic performances. ${ }^{17,31}$ Therefore, exploring a facile and convenient approach for the production of a large quantity of stable 1T TMDs with high activity and stability is imperative.

It has been well established that straining a 2D material can efficiently modify its electronic properties. ${ }^{32-35}$ Ghorbani-Asl et al. proposed that tensile strain could cause signi $\square$ cant changes in the electronic structure of TMDs and modulate the semiconductor-metal transition. ${ }^{34}$ Very recently, it was found that the conversion from $2 \mathrm{H}$ - to $1 \mathrm{~T}-\mathrm{TMD}$ is associated with strain and distortion in the resulting $1 \mathrm{~T}$ phase. ${ }^{36-38}$ Additionally, Voiry et al. and Li et al. proposed that the application of strain on TMDs can enhance the density of states near the Fermilevel and in $\square$ uence the free energy of hydrogen adsorption that facilitates the HER. ${ }^{37,39}$ The local curvature in 2D TMDs, which induces curvature strain in the TMDs, holds great promise in realizing phase engineering and changing electronic states..$^{40,41}$ Carbon nanotubes, nanosized tubular structures with 
a hexagonal network, have been considered as an effective topological and structural template for regulating the growth of conformally coated 2D TMDs with a curved structure..$^{42,43}$ Furthermore, carbon nanotubes also hold great promise as a matrix to signi $\square$ cantly improve the stability of the catalysts due to their outstanding properties such as high surface area, excellent chemical stability, and extraordinary electronic conductivity. However, there are hardly any reports on the construction of curved 2D TMDs on the surface of carbon nanotubes with an intimate interface. Furthermore, the effect of such curved 2D TMDs on the phase regulation as well as the stability engineering for HER catalysis has not been addressed.

In this study, we construct curved cobalt-doped tungsten selenide $\left(\mathrm{Co}_{\mathrm{WSe}}\right)$ nanosheets conformally coated on the surface of multiwalled carbon nanotubes (MWNTs), producing a nanosized $\mathrm{Co}_{\mathrm{WSe}} / \mathrm{MWNT}$ heterostructure for HER catalysis. Notably, the introduction of MWNTs in the heterostructure provides an opportunity to improve the content and stability of the $1 \mathrm{~T}$ phase, as well as the anti-oxidative stability of the Co$\mathrm{WSe}_{2}$. Under optimized conditions, the $\mathrm{Co}-\mathrm{WSe}_{2} / \mathrm{MWNT}$ heterostructure exhibits excellent catalytic activity and stability in both acidic and alkaline environments. The detailed study of the $\mathrm{Co}-\mathrm{WSe}_{2} / \mathrm{MWNT}$ heterostructure carried out here provides an applicable approach for the exploration of novelTMD-based catalysts for future clean energy generation.

\section{Experimental}

2.1. Chemicals and materials

All of the chemicals were used directly without further puri $\square$ cation. Selenium powder (Se, 99.99\%), sodium borohydride ( $\mathrm{NaBH}, 99 \%$ ), sodium tungstate (Na WO $\$ 2 \mathrm{H} \mathrm{O}, 30 \%$ ),

$\stackrel{4}{4}$ MWNTs $(773840$ Aldrich, $\$ 98 \%$ carbon basis, O.D. $\times$ I.D. $\times$ $L 10 \mathrm{~nm} \pm 1 \mathrm{~nm} \times 4.5 \mathrm{~nm} \pm 0.5 \mathrm{~nm} \times 3-6 \mathrm{~mm})$, tungsten selenide ( $\mathrm{WSe}_{2}, 99.99 \%$ ), and $\mathrm{Na} \square$ on solution $(5 \%)$ were purchased from Aldrich. $\mathrm{N}, \mathrm{N}$-dimethylformamide (DMF, $99.8 \%)$, cobalt acetate $\left(\mathrm{Co}\left(\mathrm{CH}_{3} \mathrm{COO}\right)_{2} \$ 4 \mathrm{H}_{2} \mathrm{O}, 99.8 \%\right)$, ethanol, potassium hydroxide $(\mathrm{KOH}, 98 \%)$ and concentrated sulfuric acid $\left(\mathrm{H}_{2} \mathrm{SO}_{4}, 98 \%\right)$ were purchased from Sinopharm Chemical Reagent Co., Ltd., People's Republic of China. The deionized (DI) water used in this study was prepared using a Millipore Milli-Q water puri $\square$ cation system (18.2 MU).

\subsection{Synthesis of $\mathrm{WSe}_{2}$}

First, $8 \mathrm{mmol}$ of Se and $8 \mathrm{mmol}$ of $\mathrm{NaBH}_{4}$ were dissolved in $50 \mathrm{~mL}$ of DMF. In order to obtain a homogeneous solution, vigorous magnetic stirring was performed for 2 hours at $60^{\circ} \mathrm{C}$. Second, $10 \mathrm{~mL}$ ( $4 \mathrm{mmol}$ ) of aqueous $\mathrm{Na}_{2} \mathrm{WO}_{4} \$ 2 \mathrm{H}_{2} \mathrm{O}$ was added dropwise into the above solution under stirring for $0.5 \mathrm{~h}$. The solution was subsequently transferred into a $100 \mathrm{~mL}$ Te $\square$ onlined stainless steel autoclave. Aler that, the autoclave was heated in a conventional oven at $200{ }^{\circ} \mathrm{C}$ for $12 \mathrm{~h}$. Aler the reaction was completed, the oven was turned off and allowed to cooldown to room temperature (RT) naturally. The as-prepared products were collected by centrifugation at $\sim 7000 \mathrm{rpm}$ and washed with DI water and ethanol 4 times and then the products were dried at $40^{\circ} \mathrm{C}$ undervacuum. Finally, toimprove the crystallinity of the samples, an annealing process was performed at $300{ }^{\circ} \mathrm{C}$ for 5 hours.

\subsection{Synthesis of Co-WSe $2, \mathrm{WSe}_{2} / \mathrm{MWNTs}, \mathrm{Co}-\mathrm{WSe} / \mathrm{MWNTs}$, $\mathrm{Co}-\mathrm{WSe} / \mathrm{rGO}$ and $\mathrm{CoSe}_{x} / \mathrm{MWNTs}$}

$\mathrm{Co}_{\mathrm{WSe}}, \mathrm{WSe}_{2} / \mathrm{MWNT}$ and $\mathrm{Co}-\mathrm{WSe}_{2} / \mathrm{MWNT}$ samples were prepared via a process similar to that used for the synthesis of WSe 2 except in the presence of additional $\mathrm{Co}\left(\mathrm{CH}_{3} \mathrm{COO}\right)_{2} \$ 4 \mathrm{H}_{2} \mathrm{O}$ and MWNTs. Speci $\square$ cally, for the Co-WSe $2,0.8 \mathrm{mmol}$ of $\mathrm{Co}\left(\mathrm{CH}_{3} \mathrm{COO}\right)_{2} \$ 4 \mathrm{H}_{2} \mathrm{O}$ was added, and for the $\mathrm{WSe}_{2} / \mathrm{MWNTs}$ and $\mathrm{Co}-\mathrm{WSe}_{2} / \mathrm{MWNTs}, 20 \mathrm{mg}$ of MWNTs or $0.8 \mathrm{mmol}$ of $\mathrm{Co}\left(\mathrm{CH}_{3} \mathrm{COO}\right)_{2} \$ 4 \mathrm{H}_{2} \mathrm{O}$ and $20 \mathrm{mg}$ of MWNTs were added. Additionally, $\mathrm{Co}-\mathrm{WSe}_{2} / \mathrm{MWNT}$ heterostructures with different contents of MWNTs ( $5 \mathrm{mg}, 20 \mathrm{mg}$ and $30 \mathrm{mg}$ ) were also prepared under the sameconditions.

For Co-WSe $2 /$ rGO, $20 \mathrm{mg}$ of reduced graphene oxide (rGO) was used in place of MWNTs. GO was prepared from natural graphite powder by a modi $\square$ ed Hummers' method at a $1: 2$ weight ratio of graphite to $\mathrm{KMnO}_{4} \cdot{ }^{24} \mathrm{For} \mathrm{CoSe}_{x} / \mathrm{MWNTs}$, a process similar to that used for preparing $\mathrm{Co}_{-} \mathrm{WSe}_{2} / \mathrm{MWNTs}$ was used, except without the addition of $\mathrm{Na}_{2} \mathrm{WO}_{4} \$ 2 \mathrm{H}_{2} \mathrm{O}$.

\subsection{Characterization}

Scanning electron microscopy (SEM) images were obtained using a JEOL $6700 \mathrm{~F}$ at an accelerating voltage of $10 \mathrm{kV}$. Transmission electron microscopy (TEM) and high resolution TEM (HRTEM) images were recorded using a JEOL 2010F microscope operating at $200 \mathrm{kV}$. X-ray diffraction (XRD) patterns were acquired using a Philips model PW1825 system with Cuka radiation (1 1 $1 / 40.15406 \mathrm{~nm})$. Raman spectra were recorded on

a LabRAM HR Raman microscope with a laser excitation wavelength of $532 \mathrm{~nm}$. Ultraviolet-visible (UV-vis) absorption spectra of the sample dispersions were recorded using a Perkin Elmer Lambda 25 spectrophotometer. Contact angle measurements were acquired using contact angle analysis equipment (JC2000C1). X-ray photoelectron spectroscopy (XPS) spectra were collected on a PHI 5600 XPS Spectrometer.

\subsection{Electrochemical measurements}

$5 \mathrm{mg}$ of catalyst was added to $0.5 \mathrm{~mL}$ of ethanol, $0.5 \mathrm{~mL}$ of deionized water and $5 \mathrm{~mL}$ of $\mathrm{Na} \square$ on solution $(5 \%)$, and the mixture was ultra-sonicated for $60 \mathrm{~min}$ to form a homogeneous ink. Then $10 \mathrm{~mL}$ of the catalyst ink was drop-cast on a glassy carbon electrode $(5 \mathrm{~mm}$ in diameter, loading density $\sim 0.25 \mathrm{mg} \mathrm{cm}^{-2}$ ) by air drying. Electrochemical analysis was done using a $\mathrm{CHI} 660 \mathrm{E}$ instrument. Linear sweep voltammetry was conducted in $0.5 \mathrm{M} \mathrm{H}_{2} \mathrm{SO}_{4}$ solution or $1 \mathrm{M} \mathrm{KOH}$ solution at a scan rate of $5 \mathrm{mV} \mathrm{s}^{-1}$, using a typical three-electrode setup. The glassy carbon electrode with the catalysts on it was used as the working electrode, a Ag/ $\mathrm{AgCl}$ (saturated $\mathrm{KCl}$ ) electrode was the reference electrode and a graphite rod was the counter electrode. Electrochemical impedance spectroscopy (EIS) was performed in the open-circuit voltage range from $10^{6}$ to $0.01 \mathrm{~Hz}$ with an AC voltage of $5 \mathrm{mV}$. The Tafel slope (b) was obtained by $\square$ tting the linear portion of the Tafel plots $(\mathrm{h} 1 / 4 b \log (J)+a)$, 
where $h$ is the overpotential and $J$ is the current density. All data were reported with $i R$ compensation and all the potentials reported here were against the reversible hydrogen electrode (RHE), i.e. eqn (1) was used.

$$
\begin{aligned}
\mathrm{E}_{\mathrm{RHE}}{ }^{1 / 4} & \mathrm{E}_{\mathrm{Ag} / \mathrm{AgCl}}+0.059 \mathrm{pH} \\
& +\mathrm{E}_{\mathrm{Ag} / \mathrm{AgCl}}\left(\mathrm{E}_{\mathrm{Ag} / \mathrm{AgCl}}{ }^{1} 1 / 4+0.198 \mathrm{~V}\right)
\end{aligned}
$$

\section{Results and discussion}

\subsection{One-pot synthesis of the $\mathrm{Co}-\mathrm{WSe} / \mathrm{MWNT}$} heterostructure

The concept for the one-pot synthesis of the Co-WSe 2 / MWNT heterostructure is illustrated in Fig. 1. Typically, comparison experiments are also conducted under identical synthetic conditions without the addition of the Co precursor and/or MWNTs (Fig. S1 and S2 $\dagger$ ). Compared with the purchased bulk $\mathrm{WSe}_{2}\left(\mathrm{~B}-\mathrm{WSe}{ }_{2}\right.$ ) (Fig. S2a $\left.\mathrm{f}\right)$, the prepared WSe $\mathrm{W}_{2}$ exhibits a $\square$ owerlike morphology with a lateral size of 200-300 nm and a thickness of 25-35 nm (Fig. S2b †). Aler the introduction of Co heteroatoms into the $\mathrm{WSe}_{2}\left(\mathrm{Co}-\mathrm{WSe}_{2}\right)$, the morphology of the $\mathrm{Co}-\mathrm{WSe}_{2}$ still retains a sheet structure but the nanosheets become smaller and severely aggregate together (Fig. S2c†). When MWNTs are incorporated, the MWNTs act as an efficient topological template for the nucleation and growth of $\mathrm{Co}-\mathrm{WSe}_{2}$ nanosheets, and $\square$ nally the $\mathrm{Co}-\mathrm{WSe}_{2}$ nanosheets are conformally coated on the surface of MWNTs with a curved structure (Co-WSe2 / MWNT heterostructure, Fig. 1 and 2).

As shown in Fig. 2a and b, small sized and highly dispersed $\mathrm{Co}-\mathrm{WSe}_{2}$ nanosheets are intimately anchored on the surface of MWNTs. In particular, the lateral size of the $\mathrm{Co}-\mathrm{WSe}_{2}$ nanosheets coated on the MWNTs is $\sim 20-40 \mathrm{~nm}$, indicating a large number of exposed edges. Notably, no individual Co-WSe 2 nanosheet or aggregate is observed, indicating that the MWNTs are an efficient topological template for the growth of highly dispersed $\mathrm{Co}_{-} \mathrm{WSe}_{2}$ nanosheets with numerous accessible sites. Energy dispersive X-ray spectroscopy (Fig. S3, ESI $\dagger$ ) clearly demonstrates the homogeneous distribution of $\mathrm{W}, \mathrm{Se}$, and $\mathrm{Co}$ throughout the MWNTs. The detailed structures of the Co$\mathrm{WSe}_{2}$ /MWNT hybrids are also examined by high-resolution transmission electron microscopy (HRTEM). As shown in

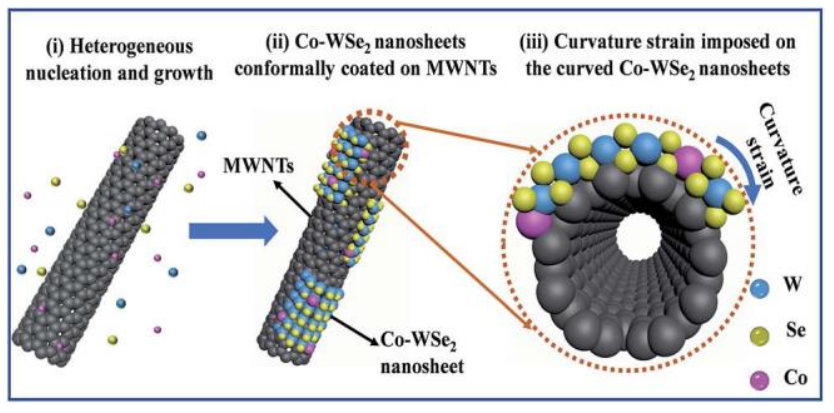

Fig. 1 Schematic illustration of the synthesis of the Co-WSez/MWNT heterostructure.

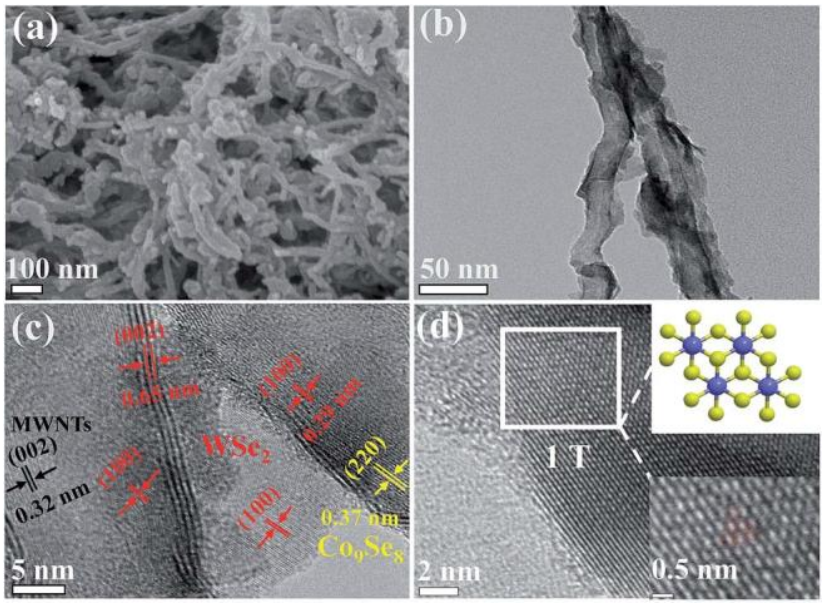

Fig. 2 Electron microscopy characterization of the Co-WSe $/$ MWNT heterostructure. (a) SEM, (b) TEM, and ( $c$ and d) HRTEM images of the $\mathrm{Co}-\mathrm{WSe}_{2} / \mathrm{MWNT}$ heterostructure. The region indicated by the square in (d) is enlarged to show the atomic structure (bottom right inset) together with the unit cells of the $1 \mathrm{~T}$ phase (top right inset).

Fig. 2c, most of the Co-WSe 2 nanosheets tightly lie across the MWNTsurface with foldededges, showing aninterlayerspacing of $0.65 \mathrm{~nm}$ and mainly comprising $2-4$ layers. In addition, the lattice spacings of $0.29 \mathrm{~nm}$ and $0.32 \mathrm{~nm}$ correspond respectively to the (100) plane of $\mathrm{WSe}_{2}$ and the interlayer spacing of the MWNTs, revealing that the small $\mathrm{Co}-\mathrm{WSe}_{2}$ nanosheets have in situ grown around the surface of the MWNTs. Notably, the presence of the lattice spacing of $0.37 \mathrm{~nm}$ indicates that some Co atoms are in the form of a Co-containing compound, i.e. CogSe8. Surprisingly, a closerlook at the image in Fig. 2 d reveals portions with hexagonal structures, which are typically seen for the 1T phase. Furthermore, more details and the associated Fast Fourier Transform (FFT) patterns of the Co-WSe 2 / MWNT heterostructure are shown in Fig. S4. $\dagger$ The corresponding diffraction patterns clearly show the polycrystalline nature of the Co$\mathrm{WSe}_{2}$ on the surface of the MWNTs, showing that many defects are involved in the crystalline planes, resulting in increased active sites. For comparison, the TEM and HRTEM images of the $\mathrm{WSe}_{2}$ and $\mathrm{Co}-\mathrm{WSe}_{2}$ samples are also provided in the ESI (Fig. S5 and S6†), and the results clearly show that the incorporation of MWNTs greatly improves the dispersibility of the catalysts, which is consistent with the $\square$ ndings from the SEM images (Fig. S2†).

A more detailed analysis of the structural information of the samples is derived from X-ray diffraction (XRD), Raman spectroscopy and ultraviolet-visible (UV-vis) absorption spectroscopy. As shown in Fig. 3a, the WSe 2 sample exhibits many prominent diffraction peaks centered at around $2 \mathrm{q} 1 / 413.62^{\circ}$, $31.41^{\circ}, 37.80^{\circ}, 47.36^{\circ}$, and $55.90^{\circ}$, matching well with the (002), (100), (103), (105), and (110) planes of WSe 2 (JCPDS card no. 381388), respectively. For the Co-WSe2 sample, we observe that the (002) peakat $2 q^{1 / 4} 13.62^{\circ}$ exhibits a sharp rise, implying that the incorporation of Co atoms tends to make the nanosheets stack together, which is coincident with the electron microscopy results. Moreover, the appearance of several new peaks marked 

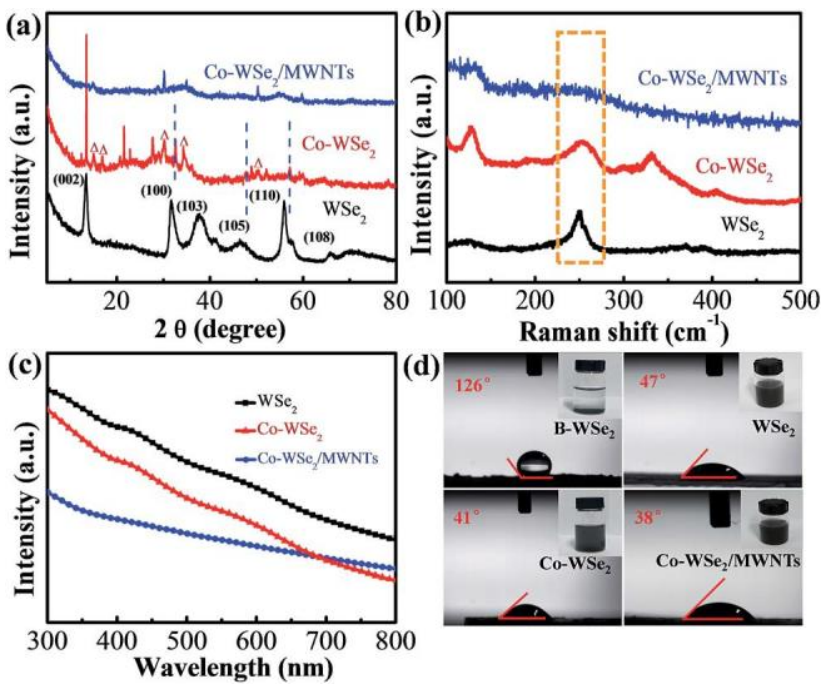

Fig. 3 XRD patterns, Raman and UV-vis absorption spectra and static contact angle images of the as-prepared samples. (a) XRD patterns, (b) Raman spectra and (c) UV-vis absorption spectra of $\mathrm{WSe}_{2}, \mathrm{Co}-\mathrm{WSe}_{2}$ and the $\mathrm{Co}-\mathrm{WSe}_{2} / \mathrm{MWNT}$ heterostructure. (d) Suspension and static

contact angle images of commercial $\mathrm{B}-\mathrm{WSe}_{2}$ and the prepared $\mathrm{WSe}_{2}$, $\mathrm{Co}-\mathrm{WSe}_{2}$ and $\mathrm{Co}-\mathrm{WSe} / \mathrm{MWNT}$ heterostructure.

with red triangles can be ascribed to $\mathrm{Co}_{9} \mathrm{Se}_{8}$ (JCPDS card no. 01089-4180); this is consistent with the HRTEM images (Fig. 2c and $\mathrm{S} 6 \dagger$ ). Besides, the positions of the (100), (105) and (110) peaks of $\mathrm{Co}^{-} \mathrm{WSe}_{2}$ are upshiped as compared to those of the $\mathrm{WSe}_{2}$ (indicated by blue dashed lines), demonstrating the successful doping of smaller Co atoms into the WSe 2 lattice. As expected, when MWNTs are incorporated, the intensity of the $\mathrm{Co}^{-W S e} 2$ diffraction peaks in the Co-WSe2/MWNT heterostructure decreases signi $\square$ cantly, indicating that the incorporation of MWNTs considerably limits the aggregation of the Co$\mathrm{WSe}_{2}$, which could lead to ample edges for high HER activity.

The as-prepared $\mathrm{WSe}_{2}, \mathrm{Co}_{-} \mathrm{WSe}_{2}$, and Co-WSe$/$ MWNT heterostructure are also characterized by Raman spectroscopy. Moreover, the Raman spectrum of the purchased B-WSe $\mathrm{W}_{2}$ is also provided (Fig. $\mathrm{S} 7 \dagger$ ) for comparison. In the Raman spectrum of the $\mathrm{B}-\mathrm{WSe} 2$, two strong characteristic peaks are observed at around $250 \mathrm{~cm}^{-1}$, assigned to the $\mathrm{E}^{2 \mathrm{~g}}$ (in-plane) and $\mathrm{A}_{1 \mathrm{~g}}$ (out-of-

plane) modes ofWSe 2 . As shown in Fig. 3b, the Co-WSe 2 /MWNT heterostructure exhibits an extremely weak Raman signal at around $250 \mathrm{~cm}^{-1}$, indicating that the heterostructure is mainly in the form of the $1 \mathrm{~T}$ phase. ${ }^{18}$ Additionally, the Co-WSe2/MWNT heterostructure exhibits two distinct peaks at around 1350 and $1590 \mathrm{~cm}^{-1}$ (Fig. S8 + ), which correspond to the D and G bands of MWNTs, respectively. Generally, the intensity ratio $I_{\mathrm{D}} / I_{\mathrm{G}}$ can be used to indicate the MWNT quality, and the $I_{\mathrm{D}} / I_{\mathrm{G}}$ value of the $\mathrm{Co}^{-W S e} \mathrm{~W}_{2} / \mathrm{MWNT}$ heterostructure $\left(I_{\mathrm{D}} / I_{\mathrm{G}} 1 / 41\right)$ indicates that there are some functional groups and defects on the surface of the MWNTs, which might act as efficient nucleation sites for the subsequent Co-WSe2 growth. ${ }^{44}$

Fig. 3c shows the UV-vis absorption spectra of $\mathrm{WSe}_{2}, \mathrm{Co}-$ $\mathrm{WSe}_{2}$, and the Co-WSe $2 / \mathrm{MWNT}$ heterostructure in water, and
400, 590 and $775 \mathrm{~nm}$ are observed for the B-WSe 2 , which are correlated with the energy splitting from the valence band spinorbit coupling in the $\mathrm{B}-\mathrm{WSe} 2 .{ }^{21,45}$ Compared with the commercial bulk WSe 2 (B-WSe2, Fig. S9†), WSe2, and $\mathrm{Co}_{2} \mathrm{WSe}_{2}$, the absorption spectrum of the Co-WSe 2 / MWNT heterostructure exhibits a monotonic change without salientabsorption bands, which is indicative of metallic behaviour. ${ }^{21}$ The optical images of the samples dispersed in water are also provided (Fig. 3d). The as-prepared samples distribute homogeneously in the water and exhibit much smaller water contact angles than that of the B-WSe2. These results clearly demonstrate that the asprepared samples, especially the Co-WSe 2 /MWNTs, possess more hydrophilic surfaces, which is also consistent with the $\square$ nding of other reported work that metallic $1 \mathrm{~T} \mathrm{MoS}_{2}$ displays hydrophilic behaviour. ${ }^{21}$

To garner further information on the atomic valence states and the composition of the samples, X-ray photoelectron spectroscopy (XPS) is carried out (Fig. 4 and S10-S13†). As shown in Fig. $4 \mathrm{a}$, deconvolution of the $\mathrm{W} 4 \mathrm{f}$ spectrum of the Co$\mathrm{WSe}_{2}$ /MWNT heterostructure yields two signals at around 32.3 and $34.3 \mathrm{eV}$ (indicated by blue curves), which are generally

attributed to $\mathrm{W}^{4+} 4 \mathrm{f}_{7 / 2}$ and $\mathrm{W}^{4+} 4 \mathrm{f}_{5 / 2}$ in the semiconducting $2 \mathrm{H}$

form, respectively. Moreover, the presence of additional peaks at lower binding energies (indicated by red curves) demonstrates the coexistence of the $1 \mathrm{~T}$ form. ${ }^{46}$ Similarly, a downshi? of binding energies is also observed for the Se $3 d_{5 / 2}$ and $\mathrm{Se} 3 \mathrm{~d}_{3} / 2$ peaks (indicated by blue and red curves in Fig. 4b). The downshilan the peak positions of $\mathrm{W} 4 \mathrm{f}$ and Se $3 \mathrm{~d}$ clearly demonstrates that we have successfully prepared the $\mathrm{Co}_{-} \mathrm{WSe}_{2} / \mathrm{MWNT}$ heterostructure with a high percentage of metallic $1 \mathrm{~T} \mathrm{WSe}_{2}$. Besides, the second set of peaks at $36.4 \mathrm{eV}$ and $38.5 \mathrm{eV}$ are assigned to $\mathrm{W}^{6+}$ (Fig. 4a), indicating partial oxidation of the Co$\mathrm{WSe}_{2}$ domain edges. ${ }^{47}$

It has been well established that the $1 \mathrm{~T}$ phase of TMDs plays a critical role in improving their electrocatalytic activity and

the UV-vis absorption spectrum of the $\mathrm{B}-\mathrm{WSe}_{2}$ is also provided in Fig. S9. $\dagger$ Three typical absorption peaks located at around 

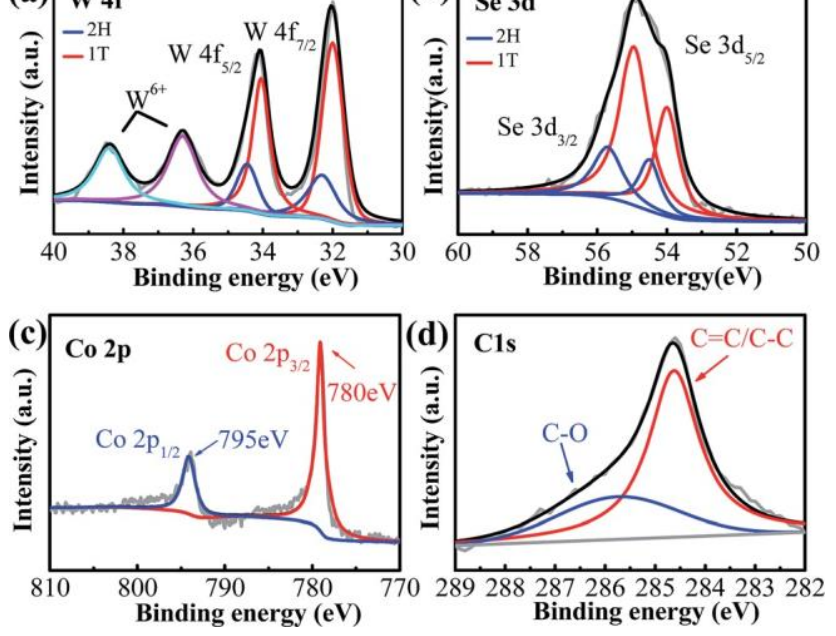

Fig. 4 High-resolution X-ray photoelectron spectroscopy (XPS) spectra of the $\mathrm{Co}-\mathrm{WSe}_{2} / \mathrm{MWNT}$ heterostructure. High resolution (a) W 4f, (b) Se 3d, (c) Co 2p, and (d) C 1s XPS spectra of the Co-WSe $/$ MWNT heterostructure. The curves are deconvoluted by Gaussian fitting. 
increasing the proportion of the $1 \mathrm{~T}$ phase can result in higher conductivity. ${ }^{48-50}$ Herein, the relative content of the $1 \mathrm{~T}$ phase in the samples is estimated from the XPS spectra and the data are summarized in Table S1. $\dagger$ A ?er introduction of the MWNTs, the content of the $1 \mathrm{~T}$ phase signi $\square$ cantly increased to $69.4 \%$ for $\mathrm{WSe}_{2} / \mathrm{MWNTs}$ and $72.1 \%$ for $\mathrm{Co}-\mathrm{WSe}_{2} / \mathrm{MWNTs}$ compared with that of the $\mathrm{WSe}_{2}(57.1 \%)$ and $\mathrm{Co}-\mathrm{WSe}_{2}$ (60.0\%). In order to validate the $1 \mathrm{~T}$ phase formation mechanism, two-dimensional (2D) reduced graphene oxide ( $\mathrm{rGO}$ ) is used as the template for the growth of $\mathrm{Co}_{-} \mathrm{WSe}_{2}$ under the same conditions, wherein the rGO can be regarded as a planar MWNT. Unsurprisingly, without the curvature strain caused by the nanosized tubular MWNTs, the $1 \mathrm{~T}$ content of $\mathrm{Co}-\mathrm{WSe}_{2} / \mathrm{rGO}$ is only $58.7 \%$ (Fig. S14†), which is much lower than that of Co-WSe 2 MWNTs (72.1\%). These pieces of experimental evidence clearly demonstrate that the curvature strain induced by the MWNTs is responsible to the formation of the predominant $1 \mathrm{~T}$ polymorph. Besides, the anti-oxidative stability of the TMD catalysts also determines their catalytic activity and the surface oxidation can severely reduce the inherent HER activity of TMDs. ${ }^{27}$ Thus, the oxidation degree of the catalysts, i.e. the relative content of $\mathrm{WO}_{3}$, is also summarized in Table $\mathrm{S} 1 . \dagger$ Clearly, the Co-WSe 2 exhibits the worst anti-oxidative stability (oxidation degree of 58.6\%). This indicates that the incorporation of the second metallic atom, $\mathrm{Co}$, into $\mathrm{WSe}_{2}$ leads to a high proportion of metal atoms being exposed on the surface, which can make them thermodynamically metastable with high surface energy and vulnerable to oxidation. Interestingly, ater the introduction of MWNTs, the oxidation degree of the $\mathrm{Co}^{-} \mathrm{WSe}_{2}$ / MWNT heterostructure decreases signi $\square$ cantly to $32.3 \%$. Based on the above results, it's easy to come to a conclusion that the introduction of MWNTs can not only increase the content of the $1 \mathrm{~T}$ phase, but also efficiently stabilize the metastable $\mathrm{Co}-\mathrm{WSe}_{2}$ against oxidative degradation.

Fig. 4c shows the Co 2p XPS spectrum of Co-WSe $2 /$ MWNTs and it can be deconvoluted into two peaks attributed to $\operatorname{Co} 2 \mathrm{p}_{3 / 2}$ and $\mathrm{Co} 2 \mathrm{p}_{1 / 2}$ at 780 and $795 \mathrm{eV}$, respectively. The separation binding energies of these two peaks close to $15 \mathrm{eV}$ can be assigned to $\mathrm{Co}^{2+}$ ions. ${ }^{51,52} \mathrm{It}$ is worth pointing out that there is nodifference between the Co $2 \mathrm{pXPS}$ spectra ofCo-WSe $\mathrm{W}_{2}$ and Co$\mathrm{WSe}_{2}$ / MWNTs, implying that the incorporation of MWNTs does not change the mode of $\mathrm{Co}_{-\mathrm{WSe}}$ (Fig. S13ct). Additionally, the C $1 \mathrm{~s}$ XPS spectrum of $\mathrm{Co}^{-W S e} \mathrm{~W}_{2} / \mathrm{MWNT}$ is shown in Fig. 4d, where the two peaks are assigned to C] C/C-C $(284.7 \mathrm{eV})$ and $\mathrm{C}-\mathrm{O}(286.0 \mathrm{eV})$ groups, respectively. ${ }^{24}$ Notably, the position of the $\mathrm{C} \ \mathrm{C} / \mathrm{C}-\mathrm{C}$ peak in the $\mathrm{Co}-\mathrm{WSe}_{2} / \mathrm{MWNT}$ heterostructure is upshi?ed by $\sim 0.2 \mathrm{eV}$ compared to the pristine MWNTs (284.5 eV, Fig. S15†), indicating efficient charge transfer from MWNTs to Co-WSe $2 .{ }^{24,42}$ Because of the oxygen-containing groups on the surface of MWNTs, the MWNTs exhibit not only a more favorable interaction with the precursor ions of Co$\mathrm{WSe}_{2}$ but also better wettability. Thus, the MWNTs could act as an efficient topological template for the nucleation and growth of curved $\mathrm{Co}_{-}-\mathrm{WSe}_{2}$ nanosheets with abundant accessible active sites. In addition, the disappearance of the carbonyl (C]O) groups at $288.1 \mathrm{eV}$ indicates that the $\mathrm{C} \mathbf{O} \mathrm{O}$ groups are mostly removed from the $\mathrm{Co}-\mathrm{WSe}_{2} / \mathrm{MWNT}$ heterostructures, which will be bene $\square$ cial for enhancing the electrical conductivity of the Co$\mathrm{WSe}_{2} / \mathrm{MWNT}$ heterostructure.

\subsection{Electrocatalytic HER activity over a wide $\mathrm{pH}$ range}

The catalytic activity of the Co-WSe 2 / MWNT heterostructure toward the HER in comparison with that of the other samples was investigated. Fig. 5 shows the HER performances of the asprepared samples and the electrocatalytic performance parameters are summarized in Table S2.† Clearly, the Co-WSe $2 /$ MWNT heterostructure exhibits the lowest overpotential $\left(174 \mathrm{mV}\right.$ ) at a current density of $-10 \mathrm{~mA} \mathrm{~cm}^{-2}$ (Fig. 5a and Table $\mathrm{S} 2 \dagger)$. For quantitative kinetic analysis of the HER activity, Tafel plots were obtained (Fig. 5b). Remarkably, the Tafel slope of

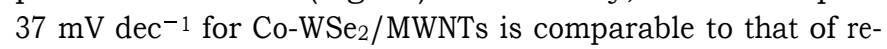
ported $\mathrm{MoS}_{2} /$ graphene $\left(\sim 41 \mathrm{mV} \mathrm{dec}^{-1}\right)^{24,53}$ and $\mathrm{Pt} / \mathrm{C}$ ( $\left.\sim 30 \mathrm{mV} \mathrm{dec}{ }^{-1}\right)$, suggesting that the Volmer-Heyrovsky HER mechanism is operative and the electrochemical desorption is the rate-limiting step. To the best of our knowledge, this Tafel slope is one of the top values for TMD-based HER catalysts reported so far (Table S3 $\dagger$ ). The effective active site area is critical for evaluating the electrocatalytic activity, and can be determined from the electrochemical double-layer capacitance $\left(C_{\mathrm{dl}}\right)$. Notably, the Co-WSe 2 /MWNT heterostructure exhibits the largest $C_{\mathrm{dl}}$ (Fig. S16†), suggesting abundant active sites for an increased HER activity.
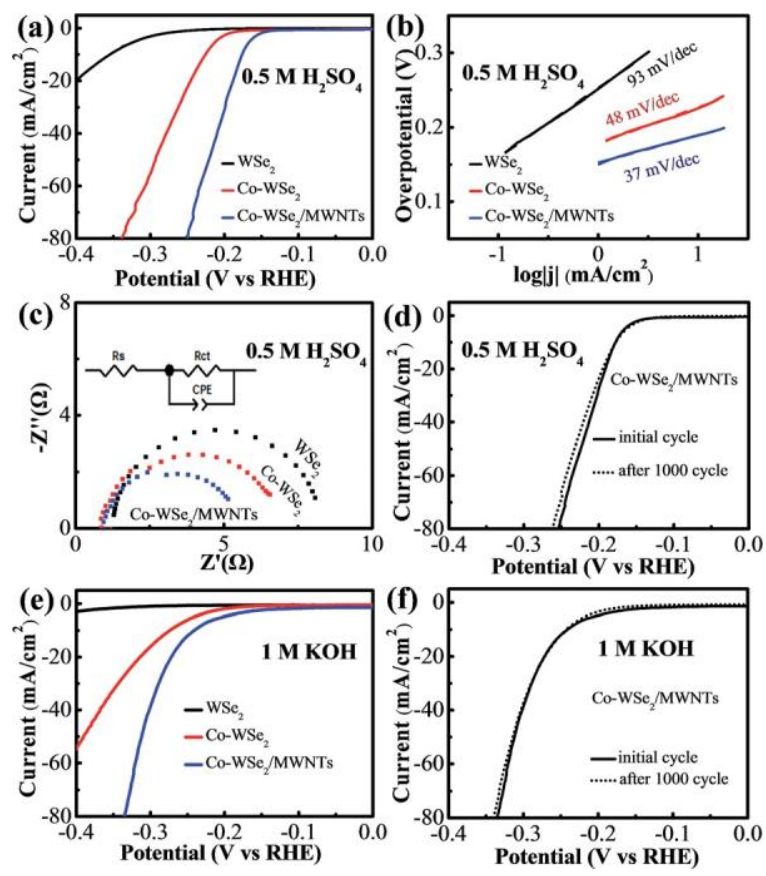

Fig. 5 Electrochemical HER measurements in acidic solution and alkaline solution, respectively. (a) Polarization curves, (b) Tafel plots, and (c) EIS Nyquist plots of WSe ${ }_{2}, \mathrm{Co}-\mathrm{WSe}_{2}$ and the $\mathrm{Co}-\mathrm{WSe}_{2} / \mathrm{MWNT}$ heterostructure in $0.5 \mathrm{M} \mathrm{H}_{2} \mathrm{SO}_{4}$. The inset in (c) is the equivalent electrical circuit. (d) Durability test for $\mathrm{Co}^{-W S e_{2}} / \mathrm{MWNTs}$ in $0.5 \mathrm{M}$ $\mathrm{H}_{2} \mathrm{SO}_{4}$. (e) Polarization curves of WSe $\mathrm{WO}_{2}, \mathrm{CSe}_{2}$ and the Co-WSe$/$ MWNT heterostructure in $1 \mathrm{M} \mathrm{KOH}$. (f) Durability test for the Co-WSe$/$ MWNT heterostructure in $1 \mathrm{MKOH}$. 
Electrochemical impedance spectroscopy (EIS) has been proved to be an efficient technique to uncover the interface reactions and electrode kinetics, and the Nyquist plots of the samples are provided in Fig. $5 \mathrm{c}$. The equivalent circuit model is shown in the inset of Fig. $5 \mathrm{c}$, and the ohmic series resistance $\left(R_{\mathrm{s}}\right)$ and charge transfer resistance $\left(R_{\mathrm{ct}}\right)$ are $\square$ tted using $Z$ simpwin solware (Table $\mathrm{S} 2 \dagger$ ). All the samples exhibit small $R_{\mathrm{s}}$, indicating good conductivity of the electrolyte. ${ }^{24}$ The charge transfer resistance, $R_{\mathrm{ct}}$, indicates the interface kinetics and a low $R_{\mathrm{ct}}$ is associated with a fast reaction rate. Obviously, the $\mathrm{Co}-\mathrm{WSe}_{2} / \mathrm{MWNT}$ heterostructure shows the lowest $R_{\mathrm{ct}}$, suggest-

ing fast HER kinetics. Furthermore, the long-term cycling stability, another signi $\square$ cant parameter of the catalysts, is investigated by a continuous long-term cycling test. As shown in Fig. 5d, the $\mathrm{Co}-\mathrm{WSe}_{2}$ / MWNT heterostructure shows negligible decay aler 1000 continuous cycles. The delamination of the catalyst might account for the slight loss of the catalytic activity. Additionally, the SEM image and Raman spectra (Fig. S17†) of the sample alder the durability test show that the morphology and compositions of the Co-WSe $/$ MWNTs show negligible changes a? structural and chemical stability of the Co-WSe 2 /MWNTs. For comparison, the cycling stability of the other samples is also measured and especially B-WSe$, \mathrm{WSe}_{2}, \mathrm{Co}-\mathrm{WSe}_{2}$ and $\mathrm{CoSe}_{x}$ MWNT catalysts exhibit larger loss of cathodic currents than that of the Co-WSe $/$ MWNT heterostructure (Fig. S18a-S22a†), indicating that the $\mathrm{Co}-\mathrm{WSe}_{2} / \mathrm{MWNT}$ heterostructure shows outstanding HER activity with long-term stability.

Besides, to investigate the effect of the MWNTs on the structure and performance of the catalysts, we prepare Co$\mathrm{WSe}_{2}$ /MWNT heterostructures with three different contents of MWNTs ( $5 \mathrm{mg}, 20 \mathrm{mg}$, and $30 \mathrm{mg}$ ). The TEM images and HER electrocatalytic activities of the Co-WSe 2 /MWNT heterostructures with different contents of MWNTs are measured (Fig. S23†). When the content of MWNTs is lower (Co-WSe $/$ MWNTs $-5 \mathrm{mg}$ ), a large number of nanosheet aggregates are observed apart from the $\mathrm{Co}-\mathrm{WSe}_{2} / \mathrm{MWNT}$ heterostructure. This leads to a relatively lower catalytic performance compared with that of the $\mathrm{Co}-\mathrm{WSe}_{2} / \mathrm{MWNTs}-20 \mathrm{mg}$, probably due to the lower efficient charge transfer from the MWNTs to $\mathrm{Co}_{-}-\mathrm{WSe}_{2}$. On the other hand, when the content of MWNTs is higher (30 mg), there are few nanosheets around the MWNTs, again leading to an inferior catalytic performance compared with that of the Co$\mathrm{WSe}_{2} / \mathrm{MWNTs}-20 \mathrm{mg}$. This mainly results from the lower loading density of the catalysts. Thus, $20 \mathrm{mg}$ of MWNTs is the optimized content for achieving the best HER performance.

Until now, most non-noble-metal HER electrocatalysts have mainly been studied only in acidic medium; nevertheless, many HER electrolyzers need active and durable catalysts that can work in alkaline medium. Thus, we also study the electrocatalytic HER activity of the samples in alkaline medium. As shown in Fig. 5e and Table S4, $\uparrow$ the Co-WSe 2 MWNT heterostructure shows outstanding activity with an overpotential of $241 \mathrm{mV}$ at a current density of $-10 \mathrm{~mA} \mathrm{~cm}^{-2}$, suggesting that the $\mathrm{Co}_{-} \mathrm{WSe}_{2}$ /MWNT heterostructure is a good candidate that can work in a wide range of $\mathrm{pH}$ values. It has been well established that Co doping of TMDs can enhance the inherent

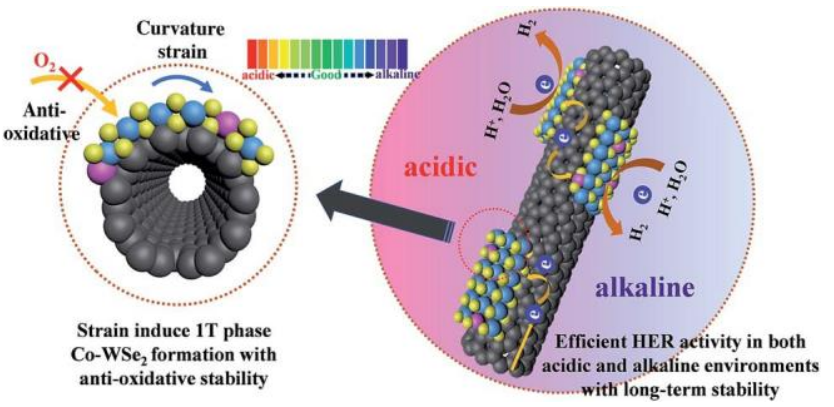

Fig. 6 HER scheme for the Co-WSe $/$ MWNT heterostructure catalyst.

activity of their active sites; nevertheless, Co-doped TMDs usually exhibit a poor activity-stability relationship and are easily affected by severe performance degradation. ${ }^{17}$ As expected, herein, the Co-WSe2 sample exhibits the worst cycling stability both in the acidic and alkaline media (Fig. S18-S22†). Conversely, the Co-WSe 2 /MWNT heterostructure exhibits superior cycling stability to the other samples (Fig. $5 \mathrm{~d}$ and f), further demonstrating that the incorporation of MWNTs signi $\square$ cantly improves the activity and stability of the catalysts.

\subsection{Mechanism investigation of the high electrocatalytic} HER activity of the Co-WSe 2 /MWNTheterostructure

For a better understanding of the underlying mechanism of the HER catalytic activity of the $\mathrm{Co}-\mathrm{WSe}_{2} / \mathrm{MWNT}$ heterostructure, a detailed HERschemeis developed based on theaboveanalysis and is shown in Fig. 6. First, the small sized tubular MWNTs act as an efficient topological template for the nucleation and growth of curved $\mathrm{Co}-\mathrm{WSe} 2$ nanosheets. They are employed to induce the formation of the protuberant $1 \mathrm{~T}$ phase Co- $\mathrm{WSe}_{2}$ and restrict the metastable 1T phase with high stability. Second, the MWNTs prevent aggregation of the catalysts and improve the thermodynamic stability of the $\mathrm{Co}-\mathrm{WSe}_{2}$ catalysts to oxidation. Third, the high conductivity of MWNTs and the intimate interface between Co-WSe 2 nanosheets and MWNTs afford rapid electron transport and transfer. Taken together, multiple synergistic optimizations of the Co-WSe 2 / MWNT heterostructure by phase and stability engineering together with nanostructuring and electronic regulation are realized for efficient electrocatalytic HER in both acidic and alkaline environments with high activity and long-term stability.

\section{Conclusions}

In summary, a facile one-pot strategy has been put forward for the synthesis of a Co-WSe 2 MWNT heterostructure. The incorporation of MWNTs can not only efficiently increase the content of the $1 \mathrm{~T}$ phase and restrict the metastable $1 \mathrm{~T}$ phase with high stability by curvature strain, but also provide an excellent conductive network for enhancing the charge transport of Co$\mathrm{WSe}_{2}$ catalysts as well as stabilize the $\mathrm{Co}-\mathrm{WSe}_{2}$ against oxidative degradation. With these outstanding advantages, the $\mathrm{Co}-\mathrm{WSe}_{2} /$ MWNT heterostructure exhibits excellent HER activity and stability in both acidic and alkaline environments. This detailed 
study of the curved Co-WSe 2 /MWNT heterostructure opens a new avenue for designing cost-effective catalysts for superior HER performance.

\section{Conflicts of interest}

There are no con $\square$ icts to declare.

\section{Acknowledgements}

This work was supported by the National Natural Science Foundation of China (No. 21773216 and 21703207), the Innovation Talents Award of Henan Province (114200510019), the China Postdoctoral Science Foundation and the Henan Province Postdoctoral Science Foundation.

\section{Notes and references}

1 X. Zou and Y.Zhang, Chem. Soc. Rev., 2015, 44, 5148-5180. 2 M. Caban-Acevedo, M. L. Stone, J. R. Schmidt, J. G. Thomas, Q. Ding, H. C. Chang, M. L. Tsai, J. H. He and S. Jin, Nat. Mater., 2015, 14, 1245-1251.

3 Y. Zheng, Y. Jiao, Y. Zhu, L. H. Li, Y. Han, Y. Chen, A. Du, M. Jaroniec and S. Z. Qiao, Nat. Commun., 2014, 5, 3783.

4 X. Huang, Z. Y. Zeng and H. Zhang, Chem. Soc. Rev., 2013, 42, 1934-1946.

5 M. Chhowalla, Z. Liu and H. Zhang, Chem. Soc. Rev., 2015, 44, 2584-2586.

6 M. Chhowalla, H. S. Shin, G. Eda, L.-J. Li, K. P. Loh and H. Zhang, Nat. Chem., 2013, 5, 263-275.

7 T. F. Jaramillo, K. P. Jørgensen, J. Bonde, J. H. Nielsen, S. Horch and I. Chorkendorff, Science, 2007, 317, 100-102.

8 B. Hinnemann, P. G. Moses, J. Bonde, K. P. Jorgensen, J. H. Nielsen, S. Horch, I. Chorkendorff and J. K. Norskov, J. Am. Chem. Soc., 2005, 127, 5308-5309.

9 H. Wang, D. Kong, P. Johanes, J. J. Cha, G. Zheng, K. Yan, N. Liu and Y. Cui, Nano Lett., 2013, 13, 3426-3433.

10 J. Kibsgaard,Z. Chen, B. N. ReineckeandT. F. Jaramillo, Nat. Mater., 2012, 11, 963-969.

11 P. D. Tran, T. V. Tran, M. Orio, S. Torelli, Q. D. Truong, K. Nayuki, Y. Sasaki, S. Y. Chiam, R. Yi, I. Honma, J. Barber and V. Artero, Nat. Mater., 2016, 15, 640-646.

12 J. Xie, H. Zhang, S. Li, R. Wang, X. Sun, M. Zhou, J. Zhou, X. W. Lou and Y. Xie, Adv. Mater., 2013, 25, 5807-5813.

13 G. Ye, Y. Gong, J. Lin, B. Li, Y. He, S. T. Pantelides, W. Zhou, R. Vajtai and P. M. Ajayan, Nano Lett., 2016, 16, 1097-1103.

14 J. Bonde, P. G. Moses, T. F. Jaramillo, J. K. Norskov and I. Chorkendorff, Faraday Discuss., 2009, 140, 219-231.

15 P. D. Tran, M. Nguyen, S. S. Pramana, A. Bhattacharjee, S. Y. Chiam, J. Fize, M. J. Field, V. Artero, L. H. Wong, J. LooandJ. Barber, Energy Environ. Sci., 2012, 5, 8912-8916.

16 G. Liu, A. W. Robertson, M. M.-J. Li, W. C. H. Kuo, M. T. Darby, M. H. Muhieddine, Y. C. Lin, K. Suenaga, M. Stamatakis, J. H. Warner and S. C. E. Tsang, Nat. Chem., 2017, 9, 810-816.

17 J. Staszak-Jirkovsky, C. D. Malliakas, P. P. Lopes, N. Danilovic, S. S. Kota, K. C. Chang, B. Genorio,
D. Strmcnik, V. R. Stamenkovic, M. G. Kanatzidis and N. M. Markovic, Nat. Mater., 2016, 15, 197-203.

18 Y. Ma, B. Liu, A. Zhang, L. Chen, M. Fathi, C. Shen, A. N. Abbas, M. Ge, M. Mecklenburg and C. Zhou, ACS Nano, 2015, 9, 7383-7391.

19 Y. Yin, Y. Zhang, T. Gao, T. Yao, X. Zhang, J. Han, X. Wang, Z. Zhang, P. Xu, P. Zhang, X. Cao, B. Song and S. Jin, Adv. Mater., 2017, 29, 1700311.

20 D. Voiry, M. Salehi, R. Silva, T. Fujita, M. Chen, T. Asefa, V. B. Shenoy, G. Eda and M. Chhowalla, Nano Lett., 2013, 13, 6222-6227.

21 X. Geng, W. Sun, W. Wu, B. Chen, A. Al-Hilo, M. Benamara, H. Zhu, F. Watanabe, J. Cui and T.-p. Chen, Nat. Commun., 2016, 7, 10672.

22 Y.Lei, S. Pakhira, K. Fujisawa, X. Wang, O. O. Iyiola, N. Perea López, A. Laura Elías, L. Pulickal Rajukumar, C. Zhou, B. Kabius, N. Alem, M. Endo, R. Lv, J. L. Mendoza-Cortes and M. Terrones, ACS Nano, 2017, 11, 5103-5112.

23 J.Yang, D. Voiry, S. J.Ahn, D. Kang, A. Y. Kim, M. Chhowalla and H. S. Shin, Angew. Chem., Int. Ed., 2013, 52, 1375113754.

24 X.Zheng, J. Xu, K. Yan, H. Wang, Z. Wang and S. Yang, Chem. Mater., 2014, 26, 2344-2353.

25 H. Tang, K. Dou, C.-C. Kaun, Q. Kuang and S. Yang, J. Mater. Chem. A, 2014, 2, 360-364.

26 D. Voiry, R. Fullon, J. Yang, C. de Carvalho Castro e Silva, R. Kappera, I. Bozkurt, D. Kaplan, M. J. Lagos, P. E. Batson, G. Gupta, A. D. Mohite, L. Dong, D. Er, V. B. Shenoy, T. Asefa and M. Chhowalla, Nat. Mater., 2016, 15, 1003-1009.

27 X. Chia, A. Y. S. Eng, A. Ambrosi, S. M. Tan and M. Pumera, Chem. Rev., 2015, 115, 11941-11966.

28 Q. H. Wang, K. Kalantarzadeh, A. Kis, J. N. Coleman and M. S. Strano, Nat. Nanotechnol., 2012, 7, 699-712.

29 D. Voiry, A. Mohite and M. Chhowalla, Chem. Soc. Rev., 2015, 44, 2702-2712.

30 D. Yang, S. J. Sandoval, W. M. R. Divigalpitiya, J. C. Irwin and R. F. Frindt, Phys. Rev. B: Condens. Matter Mater. Phys., 1991, 43, 12053-12056.

31 X. Wu, Z. Wang, M. Yu, L. Xiu and J. Qiu, Adv. Mater., 2017, 29, 1607017.

32 H. J. Conley, B. Wang, J. I. Ziegler, R. F. Haglund Jr, S. T. Pantelides and K. I. Bolotin, Nano Lett., 2013, 13, 3626-3630.

33 A. Castellanos-Gomez, R. Roldan, E. Cappelluti, M. Buscema, F. Guinea, H. S. van der Zant and G. A. Steele, Nano Lett., 2013, 13, 5361-5366.

34 M. Ghorbani-Asl, S. Borini, A. Kuc and T. Heine, Phys. Rev. B: Condens. Matter Mater. Phys., 2013, 87, 235434.

35 S. B. Desai, G. Seol, J. S. Kang, H. Fang, C. Battaglia, R. Kapadia, J. W. Ager, J. Guo and A. Javey, Nano Lett., 2014, 14, 4592-4597.

36 X. Wang, X. Shen, Z. Wang, R. Yu and L. Chen, ACS Nano, 2014, 8, 11394-11400.

37 D. Voiry, H. Yamaguchi, J. Li, R. Silva, D. C. Alves, T. Fujita, M. Chen, T. Asefa, V. B. Shenoy, G. Eda and M. Chhowalla, Nat. Mater., 2013, 12, 850-855. 
38 Y.Qi, Q.Xu, Y.Wang, B.Yan, Y. RenandZ.Chen, ACSNano, 2016, 10, 2903-2909.

39 H. Li, C. Tsai, A. L. Koh, L. Cai, A. W. Contryman, A. H. Fragapane, J. Zhao, H. S. Han, H. C. Manoharan, F. Abild-Pedersen, J. K. Norskov and X. Zheng, Nat. Mater., 2016, 15, 48-53.

40 I. Miloševic̄, B. Nikoliç, E. Dobardžic̄, M. Damnjanović, I. Popov and G. Seifert, Phys. Rev. B: Condens. Matter Mater. Phys., 2007, 76, 233414.

41 X.H.Wang, C.C.ZhengandJ.Q.Ning, Sci. Rep., 2016, 6,33091. 42 Q. Liu, Q. Fang, W. Chu, Y. Wan, X. Li, W. Xu, M. Habib, S. Tao, Y. Zhou, D. Liu, T. Xiang, A. Khalil, X. Wu, M. Chhowalla, P. M. Ajayan and L. SongL, Chem. Mater., 2017, 29, 4738-4744.

43 L. Ma, W.-X. Chen, Z.-D. Xu, J.-B. Xia and X. Li, Nanotechnology, 2006, 17, 571-574.

44 L. Yang, S. Jiang, Y. Zhao, L. Zhu, S. Chen, X. Wang, Q. Wu, J. Ma, Y. Ma and Z. Hu, Angew. Chem., Int. Ed., 2011, 50, 7132-7135.
45 K. F. Mak, C. Lee, J. Hone, J. Shan and T. F. Heinz, Phys. Rev. Lett., 2010, 105, 136805.

46 A. Ambrosi, Z. Sofer and M. Pumera, Chem. Commun., 2015, 51, 8450-8453.

47 J. Guo, Y. Shi, X. Bai, X. Wang and T. L. Ma, J. Mater. Chem. A, 2015, 3, 24397-24404.

48 F. Wang, J. Li, F. Wang, T. A. Shifa, Z. Cheng, Z. Wang, K. Xu, X.Zhan, Q. Wang, Y. Huang, C. Jiang and J. He, Adv. Funct. Mater., 2015, 25, 6077-6083.

49 M. A. Lukowski, A. S. Daniel, F. Meng, A. Forticaux, L. Li and S. Jin, J. Am. Chem. Soc., 2013, 135, 10274-10277.

50 M. A. Lukowski, A. S. Daniel, C. R. English, F. Meng, A. Forticaux, R. J. Hamers and S. Jin, Energy Environ. Sci., 2014, 7, 2608-2613.

51 X. Yang, H. Sun, P. Zan, L. Zhao and J. Lian, J. Mater. Chem. A, 2016, 4, 18857-18867.

52 Y. Li, X. Li, J. Wang, Y. Jun and Z. Tang, RSC Adv., 2017, 7, 2919-2925.

53 Y. Li, H. Wang, L. Xie, Y. Liang, G. Hong and H. Dai, J. Am. Chem. Soc., 2011, 133, 7296-7299. 